\title{
SEVERE INFECTIONS DURING THE TREATMENT OF CHILDREN WITH ACUTE LYMPHOBLASTIC LEUKEMIA
}

D. Doganis, A. Pourtsidis, M. Servitzoglou, K. Kapetaniou, M. Nikita, S. Papachristidou, E. Magkou, M. Baka "P \& A Kyriakou" Children's Hospital, Oncology Department, Athens, Greece

Aim: To describe the incidence and type of severe infections (SIs) during the treatment of children with Acute Lymphoblastic Leukemia (ALL).

Patients - Methods: We evaluated the charts of 77 children (44 boys, 33 girls, median age 5.4 yo, range 1-16 yo, Standard Risk-SR:7, Intermediate Risk-IR:50, High Risk-HR:20) with ALL treated from $01 / 2013$ to $12 / 2017$ and we recorded the episodes of SIs during their treatment.

\section{$\underline{\text { Results }}$}

Serious infections were seen in 36 patients (median age 5.9 yo). A total number of 54 Sls were recorded (in 11 patients, more than 1 episode), table 1 . The incidence of at least one SI was found to be $16 / 44$ (36\%) among boys and 20/33 among girls (61\%), $p=0.04$, whereas among SR, IR and HR patients was $2 / 7(29 \%), 24 / 50(48 \%)$ and $10 / 20(50 \%)$ respectively, $p=n s$ (table 2,3). Regarding age, no significant differences were detected. Most of the infection episodes (31) were recorded during the induction treatment ( $n=148$ cycles). During the consolidation $(n=216)$, re-induction $(n=116)$ and maintenance phase $(n=42)$ were recorded 2,8 and 5 episodes respectively whereas during the high-risk protocols $(n=81)$ were detected 8 cases (table 4). Regarding the infection type, we detected 37 (69\%) episodes of Blood Stream Infection (BSI), 7 of severe local infection, influenza:2, fungemia:1, lung aspergillosis:2, herpes zoster:4 and 1 episode of appendicitis (table 5).

\section{Table 1. number of episodes per patient (total $=54$ )}

\section{5 pts}

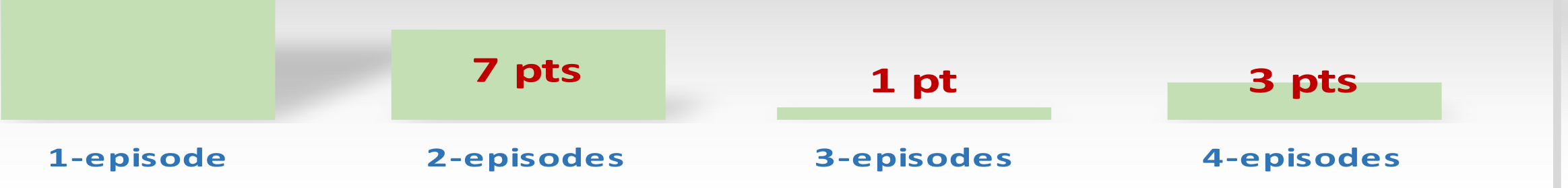

$100 \%$

$80 \%$

$60 \%$

$40 \%$

$20 \%$

$0 \%$

$100 \%$
$80 \%$
$60 \%$
$40 \%$
$20 \%$
$0 \%$
Table 2. Episodes per gender

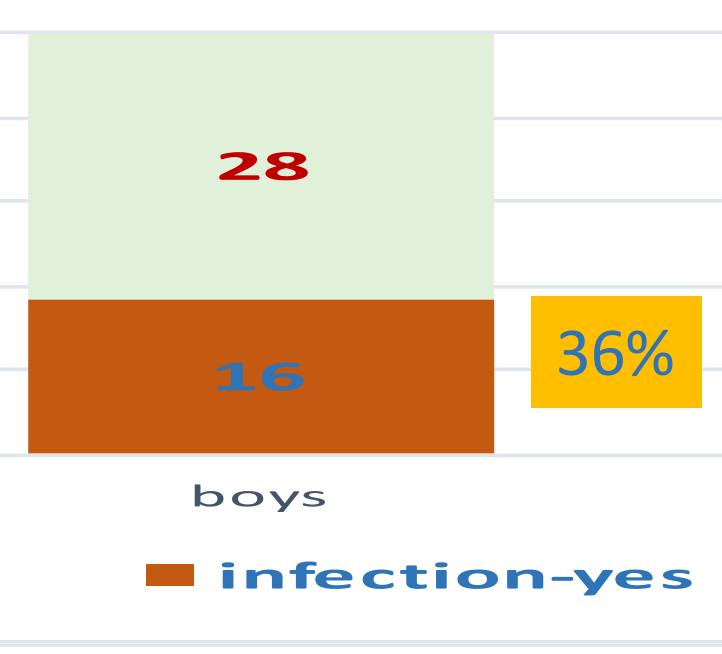

$p=0.04$ infection-yes

infection-no

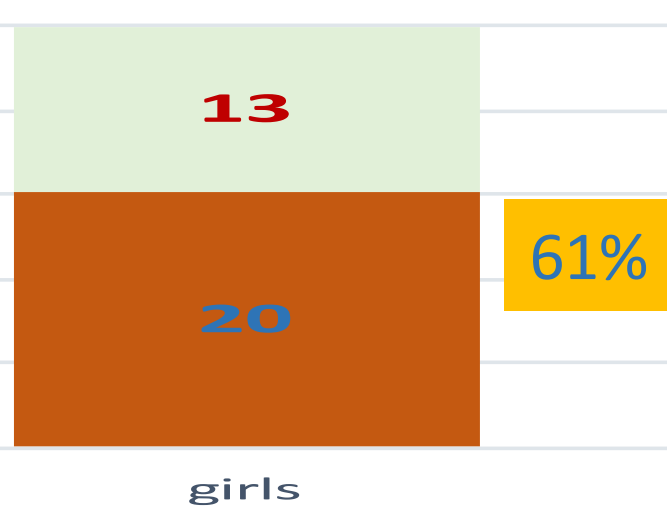

girls
Table 3. Episodes per Risk Group

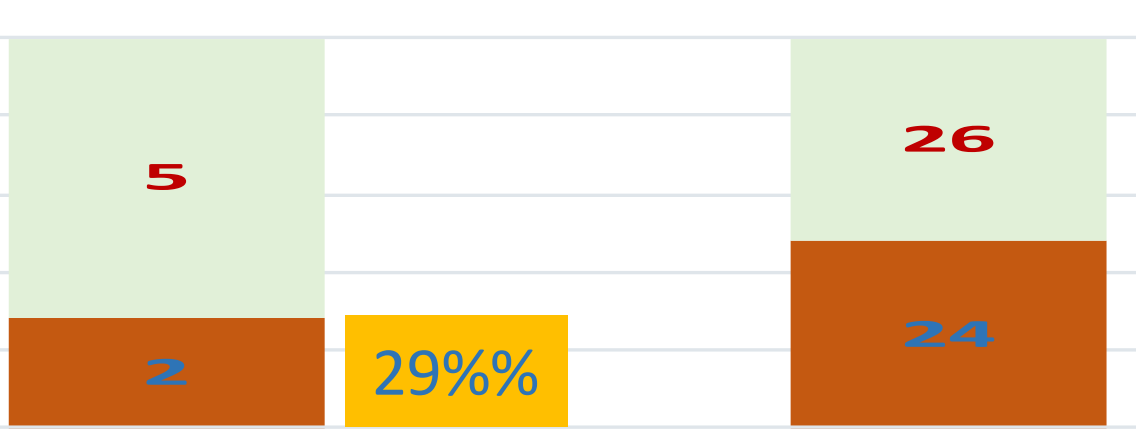

$48 \%$

- infection-yes

infection-no

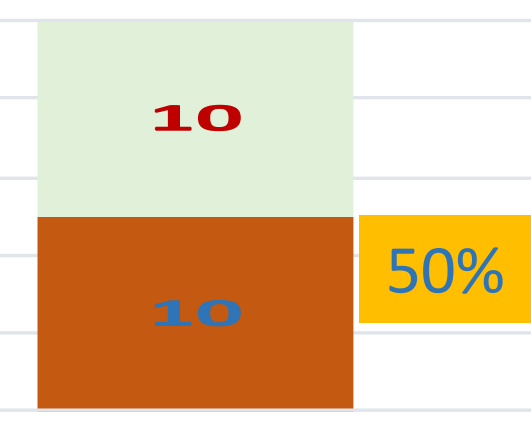

HR
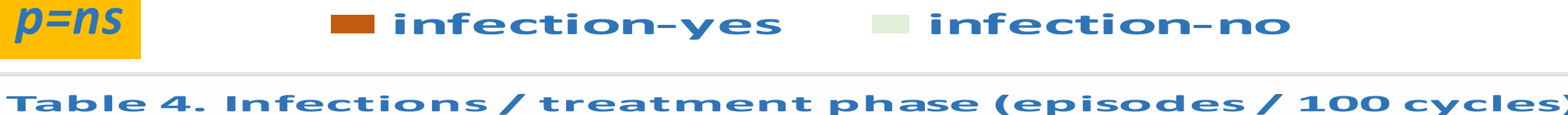

20,0

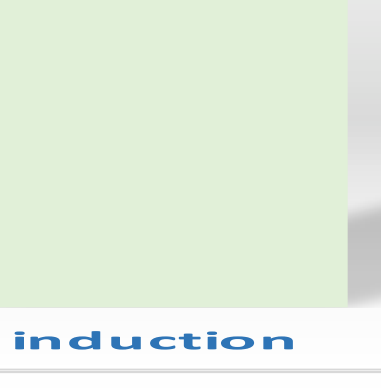

Table 5. Type of infection

HERPES

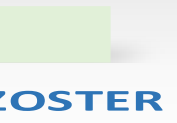

LUNG
ASPERGILLOSIS

NGEMIA

APPENDICITIS 